\title{
Efficient and precise base editing in rabbits using human APOBEC3A-nCas9 fusions
}

\author{
Zhiquan Liu', Siyu Chen', Huanhuan Shan', Quanjun Zhang'², Mao Chen', Liangxue Lai ${ }^{1,2,3,4}$ and Zhanjun Li ${ }^{1}$
}

\section{Dear Editor:}

The largest class of known human genetic diseases are caused by point mutations or single-nucleotide polymorphisms $(\mathrm{SNPs})^{1}$. Base editors that can achieve targeted C-to- $\mathrm{T}$ conversions without generating DNA double-strand breaks, or requiring a donor template, represent significant advances in both disease modeling and gene therapy ${ }^{2}$. However, the currently used base editor 3 (BE3), derived from the rAPOBEC1 (rA1)-nCas9 (Cas9 nickase, D10A) fusion, is limited by low editing efficiency in GC contexts, and by high bystander activity when more than one $C$ is present in the editing window ${ }^{2,3}$. Recently, human APOBEC3A (hA3A) was substituted for rA1 to produce new base editors (Fig. 1a). The editing window for hA3A-BEs was expanded to $\sim 12 \mathrm{nt}$ in human cells, and $\sim 17 \mathrm{nt}$ in plants and editing efficiency increased in GC contexts ${ }^{4,5}$. An engineered hA3A variant, hA3AY130F, had a limited editing window, similar to that of BE3 ( 6 nt in human cells) $)^{4,6}$. Another variant, hA3AN57G, showed a striking preference for the TCR (A/G) motif and a reduction in bystander mutations ${ }^{7}$. In this study, we demonstrate the effectiveness of the hA3AnCas9 fusion and its associated variants (Y130F and N57G) to induce efficient and precise base editing in rabbits.

We first tested hA3A-eBE in rabbit embryos at three target sites where GC (Tyr-1 and Tyr-3) or non-GC (Tyr2) contexts are contained in the editing window (Fig. 1b).

\footnotetext{
Correspondence: Liangxue Lai (lai_liangxue@gibh.ac.cn) or

Zhanjun Li (lizj_1998@jlu.edu.cn)

${ }^{1}$ Key Laboratory of Zoonosis Research, Ministry of Education, College of Animal Science, Jilin University, Changchun 130062, China

${ }^{2}$ CAS Key Laboratory of Regenerative Biology, Guangdong Provincial Key Laboratory of Stem Cell and Regenerative Medicine, South China Institute for Stem Cell Biology and Regenerative Medicine, Guangzhou Institutes of Biomedicine and Health, Chinese Academy of Sciences, Guangzhou 510530, China

Full list of author information is available at the end of the article.

These authors contributed equally: Zhiquan Liu, Siyu Chen
}

As observed in human cells and plants ${ }^{4,5}$, hA3A-eBE showed a significantly expanded base editing window $(\sim 20 \mathrm{nt})$, compared with that of BE3 $(\sim 8 \mathrm{nt})$, and a higher editing efficiency in the GC context at Tyr-1 and Tyr-3 in rabbit blastocysts (Fig. 1c; Supplementary Figs. S1-3). Moreover, the formation of by-products including indels and non-C-to-T conversions by hA3A-eBE was slightly reduced compared to that by BE3 (Supplementary Fig. S4). These results indicated that hA3A-eBE can induce efficient $\mathrm{C}$-to- $\mathrm{T}$ base editing with a large editing window and, independently of context, it could increase genometargeting scope ${ }^{8}$.

Next, we examined the ability of hA3A-eBE-Y130F (narrowed editing window ${ }^{4}$ ) to perform base editing in rabbit embryos. A total of six single guide RNAs (sgRNA) containing GC and non-GC contexts were selected to evaluate the editing efficiency of hA3A-eBE-Y130F (Fig. $1 \mathrm{~b})$. The results showed that the primary deamination window for hA3A-eBE-Y130F spanned $7 \mathrm{nt}$ (from protospacer positions 3 to 9), and is similar to that of BE3 in rabbit embryos (Supplementary Figs. S1-3 and S5-8). The genomic DNA was edited at all target sites, with the efficiency of hA3A-eBE-Y130F being slightly higher than that of $\mathrm{BE} 3(81.11 \pm 2.72 \%$ versus $75.33 \pm 4.02 \%)$ in nonGC contexts (Fig. 1d, e), consistent with reported findings in human cells and mice ${ }^{4,6}$. Within the editing window, in GC contexts, hA3A-eBE-Y130F induced significantly higher editing efficiencies than BE3 $(63.00 \pm 4.82 \%$ versus $28.44 \pm 4.96 \%, p<0.0001$ ) (Fig. 1f, g). At all target sites, hA3A-eBE-Y130F-induced non-C-to-T conversions were significantly reduced $(0.00 \pm 0.00 \%$ versus $10.32 \pm 3.02 \%$, $p<0.01$ ), but not indels, when compared with BE3 (Fig. $1 \mathrm{~h}, \mathrm{i})$. The average frequency of by-products is relatively low using BE3, but in some samples, it reached $40-50 \%$. This is consistent with the relatively high by-product frequency in individual mice and rabbit embryos using BE3 in previous studies, ${ }^{6-11}$. Overall, these results 


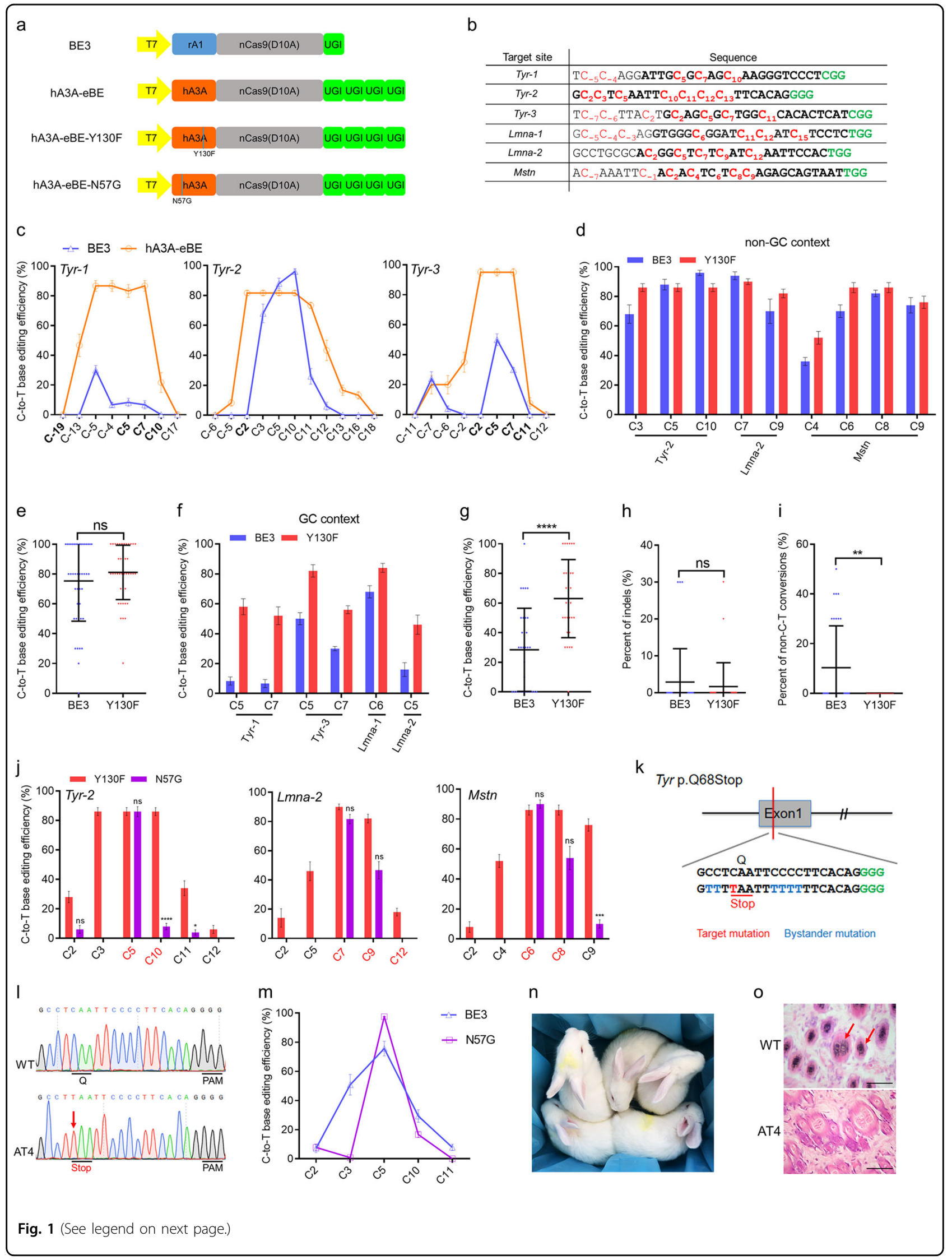


(see figure on previous page)

Fig. 1 hA3A-nCas9 fusions induce efficient and precise C-to-T base editing in vivo. a Schematic representation of BE3 and hA3A-nCas9 fusion architecture. $\mathbf{b}$ Target-site sequences used in this study. Target sequence (black), sgRNA (bold), PAM region (green), target Cs (red, counting the PAM as positions 21-23). WT, wild-type. c Frequencies of single C-to-T conversions using BE3 and hA3A-eBE at three sites in rabbit embryos. GC contexts are indicated in bold on the $x$ axis. $\mathbf{d}$, $\mathbf{f}$ Comparison of C-to-T editing efficiency induced by BE3 and hA3A-eBE-Y130F in the target sites in non-GC context (d) or GC context (f). e, g Statistical analysis of the C-to-T editing frequency induced by BE3 and hA3A-eBE-Y130F in (d), (f), respectively. Fortyfive target Cs for BE3 and 45 target Cs for hA3A-eBE-Y130F (e). Thirty-two target Cs for BE3 and 30 target Cs for hA3A-eBE-Y130F (g). h, i Statistical analysis of indels (h) or non-C-to-T conversions (i) induced by BE3 and hA3A-eBE-Y130F in rabbit embryos. Thirty-one embryos for BE3 and 30 embryos for hA3A-eBE-Y130F. $\mathbf{j}$ Frequencies of targeted single C-to-T conversions introduced by hA3A-eBE-Y130F and hA3A-eBE-N57G at three target sites in rabbit embryos. The TC contexts are indicated in red on the $x$ axis. $\mathbf{k}$ The target sequence at the Tyr p. Q68Stop locus. The PAM and sgRNA target sequences are shown in green and black, respectively. Target mutation (red), bystander mutation (blue). I Representative Sanger sequencing chromatograms from a WT, and mutant rabbit (AT4). The red arrow indicates the substituted nucleotide. The relevant codon identities at the target sites are presented beneath the DNA sequence. $\mathbf{m}$ Comparison of the editing efficiency of single C-to-T conversions between BE3 and hA3A-eBEN57G, at Tyr Q68Stop in F0 rabbit. The efficiency data of BE3 comes from our previous report ${ }^{11}$. $\mathbf{n}$ All five F0 rabbits exhibited a systemic albino phenotype. o H\&E staining of skin from WT and Tyr mutant (AT4) rabbits. The red arrow highlights the melanin in the basal layer of the epidermis. Scale bars: $50 \mu \mathrm{m}$

demonstrated that hA3A-eBE-Y130F could induce efficient $\mathrm{C}$-to- $\mathrm{T}$ base editing in rabbit embryos with high product purity, independently of sequence context.

The editing activities of an engineered base editor, hA3A-N57G, was high on cytidines in TCR (A/G) motifs, but greatly reduced when cytidines are in other sequence contexts $^{7}$. Thus, to further improve precision in the TC context, a N57G mutation was introduced into hA3A-eBE to create hA3A-eBE-N57G (Fig. 1a). Three sgRNAs containing both TC and non-TC contexts were then selected for testing (Fig. 1b, j). The editing window for hA3A-eBEN57G spanned 5-9 nt, which is similar to that observed in human cells ${ }^{7}$, but the editing efficiency was significantly reduced in adjacent non-TC contexts, at all tested sites (Fig. 1j).

It is known that the Tyr gene is the major causal gene of human oculocutaneous albinism $(\mathrm{OCA})^{12}$. The high frequency of bystander mutations (86\%) was determined in the founder (F0) rabbits using BE3 in our previous study $^{11}$. Thus, to generate a rabbit model to precisely mimic OCA, the mRNA encoding hA3A-eBE-N57G and sgRNA target to Tyr p.Q68Stop were injected into zygotes, making desired mutations in the TC context adjacent to multiple Cs (Fig. 1k). Consequently, five pups were obtained after injection and transplantation (Supplementary Table S1). The genotypes of the pups were determined using Sanger sequencing and deep sequencing. The results showed that all of them $(100 \%)$ carried homozygous p.Q68Stop mutation (Figs. 11 and S9). Strikingly, hA3A-eBE-N57G significantly reduced bystander mutations of Tyr p.Q68Stop mutation in a TC context compared with that of the BE3 system (28\% versus $86 \%$ ) in founder rabbits (Supplementary Table S1). Further statistical analysis demonstrated the efficiency and precision of the hA3A-eBE-N57G-mediated mutation of Tyr p.Q68Stop, which has significantly fewer bystander mutations than does the BE3 system (target $\mathrm{C} 5$ on TC context, $97.60 \pm 0.40 \%$ versus $75.67 \pm 12.25 \%$; bystander C3 in CC context, $0.80 \pm 0.49 \%$ versus $50.83 \pm 17.33 \%$ ) (Fig. $1 \mathrm{~m}$ ). Moreover, a low proportion of indels or non-Cto- $\mathrm{T}$ mutations were detected in the F0 rabbits (Supplementary Fig. S9), consistent with a previous study using $\mathrm{BE}^{11}$. Consistent with their genotypes, all five F0 rabbits exhibited a completely albino phenotype (Fig. 1n). Furthermore, hematoxylin and eosin (H\&E) staining revealed the absence of melanin in the skin of mutant rabbits, compared with WT rabbits (Fig. 10). In addition, deep sequencing was carried out to evaluate the off-target effect of the representative AT4 mutant. No obvious offtarget mutations were detected at potential off-target sites (Supplementary Fig. S10), demonstrating that the hA3AeBE-N57G system can mediate efficient base editing on the TCR (A/G) motif with high specificity in vivo.

In summary, we demonstrated that hA3A-eBE can induce efficient $\mathrm{C}$-to- $\mathrm{T}$ conversions with a large editing window, and that hA3A-eBE-Y130F, having a narrowed editing window, can function as a generic version of the base editor, particularly at pathogenic SNPs in GC contexts. Furthermore, hA3A-eBE-N57G can induce efficient base editing in the TC context and minimize bystander mutations in vivo. Thus, these three APOBEC3A-nCas9 base editors expand the scope and improve the precision of the currently used BE3 system.

\section{Acknowledgements}

The authors thank Peiran Hu and Nannan Li at the Embryo Engineering Center for critical technical assistance. This study was financially supported by the National Key Research and Development Program of China Stem Cell and Translational Research (2017YFA0105101), the Program for Changjiang Scholars and Innovative Research Team in University (No.IRT_16R32), the Strategic Priority Research Program of the Chinese Academy of Sciences (XDA16030501, XDA16030503), and Key Research \& Development Program of Guangzhou Regenerative Medicine and Health Guangdong Laboratory (2018GZR110104004). 


\section{Author contributions}

Z.L., L.L., and Z.L. conceived and designed the experiments. Z.L. and S.C. performed the experiments. Z.L., S.C., H.S. analyzed the data. Q.Z. and M.C. contributed reagents/materials/analysis tools. Z.L. and Z.L. wrote the paper. All authors have read and approved the final manuscript.

\section{Author details}

${ }^{1}$ Key Laboratory of Zoonosis Research, Ministry of Education, College of Animal Science, Jilin University, Changchun 130062, China. ${ }^{2}$ CAS Key Laboratory of Regenerative Biology, Guangdong Provincial Key Laboratory of Stem Cell and Regenerative Medicine, South China Institute for Stem Cell Biology and Regenerative Medicine, Guangzhou Institutes of Biomedicine and Health, Chinese Academy of Sciences, Guangzhou 510530, China. ${ }^{3}$ Guangzhou Regenerative Medicine and Health Guang Dong Laboratory (GRMH-GDL), Guangzhou 510005, China. ${ }^{4}$ Institute for Stem Cell and Regeneration, Chinese Academy of Sciences, Beijing 100101, China

\section{Conflict of interest}

The authors declare that they have no conflict of interest.

\section{Publisher's note}

Springer Nature remains neutral with regard to jurisdictional claims in published maps and institutional affiliations.

Supplementary Information accompanies the paper at (https://doi.org/ 10.1038/s41421-019-0099-5).

Received: 22 January 2019 Revised: 20 April 2019 Accepted: 22 April 2019 Published online: 11 June 2019

\section{References}

1. Landrum, M. J. et al. ClinVar: improving access to variant interpretations and supporting evidence. Nucleic Acids Res. 46, D1062-d1067 (2018).

2. Komor, A. C., Kim, Y. B., Packer, M. S., Zuris, J. A. \& Liu, D. R. Programmable editing of a target base in genomic DNA without double-stranded DNA cleavage. Nature 533, 420-424 (2016)

3. Kim, Y. B., Komor, A. C. \& Levy, J. M. Increasing the genome-targeting scope and precision of base editing with engineered Cas9-cytidine deaminase fusions. Nat. Biotechnol. 35, 371-376 (2017).

4. Wang, X., Li, J., Wang, Y. \& Yang, B. Efficient base editing in methylated regions with a human APOBEC3A-Cas9 fusion. Nat. Biotechnol. 36, 946-949 (2018).

5. Zong, Y. et al. Efficient C-to-T base editing in plants using a fusion of nCas9 and human APOBEC3A. Nat. Biotechnol. 36, 950-953 (2018).

6. $L i$, J. et al. Efficient base editing in $\mathrm{G} / \mathrm{C}$-rich regions to model androgen insensitivity syndrome. Cell Res. 29, 174-176 (2019).

7. Gehrke, J. M., Cervantes, O. \& Clement, M. K. An APOBEC3A-Cas9 base editor with minimized bystander and off-target activities. Nat. Biotechnol. 36, 977-982 (2018)

8. Billon, P. et al. CRISPR-mediated base editing enables efficient disruption of eukaryotic genes through induction of STOP codons. Mol. Cell 67, 1068-1079. e1064 (2017)

9. Kim, K. et al. Highly efficient RNA-guided base editing in mouse embryos. Nat. Biotechnol. 35, 435-437 (2017).

10. Liang, P. et al. Effective gene editing by high-fidelity base editor 2 in mouse zygotes. Protein Cell 8, 601-611 (2017).

11. Liu, Z. et al. Highly efficient RNA-guided base editing in rabbit. Nat. Commun. 9, 2717 (2018).

12. Oetting, W. S. \& King, R. A. Molecular basis of albinism: mutations and polymorphisms of pigmentation genes associated with albinism. Hum. Mutat. 13 99-115 (1999). 